\title{
Interdiffusion and magnetism of the Co/Rh interface studied by magnetization induced second harmonic generation
}

\author{
F. Manders, K. J. Veenstra, A. Kirilyuk, and Th. Rasinga) \\ Research Institute for Materials, University of Nijmegen, Toernooiveld 1, 6525 ED Nijmegen, \\ The Netherlands \\ H. A. M. van den Berg \\ Siemens AG, D-91052 Erlangen, Germany \\ N. Persat \\ IPCMS-GEMME, UMR 46 CNRS-ULP, 23 rue du Loess 67037 Strasbourg, France
}

(Received 24 October 1997; accepted for publication 12 October 1998)

The nonlinear magneto-optical response from a $\mathrm{Rh} / \mathrm{Co} / \mathrm{Cu}$ multilayer shows a strong temperature dependence which can be directly related to changes at the $\mathrm{Co} / \mathrm{Rh}$ interface. The activation energy for these changes can be extracted by in situ magnetization induced second harmonic generation measurements during annealing. (C) 1998 American Institute of Physics. [S0003-6951(98)03250-1]

Some of the most exciting discoveries in magnetic multilayer systems are the observation of the oscillatory exchange coupling between two ferromagnetic layers as a function of a nonmagnetic spacer layer ${ }^{1,2}$ and the closely related effect of giant magneto-resistance (GMR). ${ }^{3}$ As the interfaces between these layers appear to play an essential role for these phenomena and consequently for the device properties based on them, detailed studies of the magnetic properties of the interfaces are required. Probing of the buried interfaces in a direct way is very challenging, especially with a nondestructive method.

Magnetization induced second harmonic generation (MSHG) is a new nonlinear magneto-optical technique that combines interface sensitivity with huge magneto-optical effects. ${ }^{4-6}$ These effects are due to the simultaneous breaking of inversion symmetry (at interfaces) and time-reversal symmetry $^{7}$ (by the magnetization).

In this letter we show how this new nonlinear magnetooptical technique can be used to probe the (changes in the) magnetic $\mathrm{Co} / \mathrm{Rh}$ interface properties. MSHG allows in situ probing of the buried $\mathrm{Co} / \mathrm{Rh}$ interface during annealing. By plotting the thus obtained change in the interface magnetization in an Arrhenius plot, we obtained the activation energy for the changes at the $\mathrm{Co} / \mathrm{Rh}$ interface. We speculate that these changes are directly related to the interdiffusion of Co into Rh.

The optical second harmonic polarization $\mathbf{P}(2 \omega)$ of a magnetic medium is generally described by a third rank polar tensor $\chi_{i j k}^{\mathrm{cr}}$ for the crystallographic contribution and a fourth rank axial tensor $\chi_{i j k l}^{\text {magn }}$ for the magnetization induced part

$$
P_{i}(2 \omega)=\chi_{i j k}^{\mathrm{cr}} E_{j}(\omega) E_{k}(\omega)+\chi_{i j k l}^{\mathrm{magn}} E_{j}(\omega) E_{k}(\omega) M_{1},
$$

where $\mathbf{E}(\omega)$ is the incoming light field and $\mathbf{M}$ is the magnetization of the medium. In centrosymmetric materials, both tensors are only nonzero at surfaces and interfaces, where the inversion symmetry is broken. For a particular magnetooptical configuration, e.g., polar or transversal (and for a

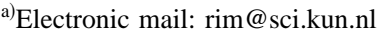

high-symmetry surface), one may simplify Eq. (1) by one third rank tensor with different components, that are either even or odd in $\mathbf{M}$, describing the crystallographic or the magnetization induced contribution, ${ }^{8}$ respectively

$$
\mathbf{P}(2 \omega, \pm \mathbf{M})=\left(\vec{\chi}_{\text {even }}^{(2)}( \pm \mathbf{M}) \pm \vec{\chi}_{\text {odd }}^{(2)}( \pm \mathbf{M})\right) E^{2}(\omega) .
$$

In the transversal geometry both $\vec{\chi}_{\text {even }}^{(2)}$ and $\vec{\chi}_{\text {odd }}^{(2)}$ act along the same direction, and Eq. (2) shows a change in the absolute value of $\mathbf{P}(2 \omega)$, resulting in a change in the SH intensity $I_{\mathrm{SH}} \propto|P(2 \omega)|^{2}$. For that case the asymmetry of the MSHG signal can be defined as

$$
A\left(q_{\text {in }} q_{\text {out }}\right)=\frac{I\left(2 \omega, q_{\text {in }} q_{\text {out }}, M^{+}\right)-I\left(2 \omega, q_{\text {in }} q_{\text {out }}, M^{-}\right)}{I\left(2 \omega, q_{\text {in }} q_{\text {out }}, M^{+}\right)+I\left(2 \omega, q_{\text {in }} q_{\text {out }}, M^{-}\right)},
$$

where $I\left(2 \omega, q_{\text {in }} q_{\text {out }}, M^{+}\right)$and $I\left(2 \omega, q_{\text {in }} q_{\text {out }}, M^{-}\right)$are the $q_{\text {in }} q_{\text {out }}$ polarized second harmonic (SH) intensities for opposite direction of the magnetization. Here, in contrast with MOKE, the average intensity $I_{\mathrm{SH}}$ is also an important parameter directly representing the electronic structure of an interface or a thin film.

The annealing of the samples will cause a change at the $\mathrm{Co} / \mathrm{Rh}$ interface. This will affect the magnetization at the interface, and consequently $\chi_{\text {odd }}^{(2)}$. Because structural changes will affect both $\chi_{\text {even }}^{(2)}$ and $\chi_{\text {odd }}^{(2)}$, a sensitive way to probe the magnetic changes at the $\mathrm{Co} / \mathrm{Rh}$ interface is to monitor the asymmetry, $A$, as a function of time and temperature.

The sample consisted of a $50 \mathrm{~nm}$ Co film covered with a $2 \mathrm{~nm}$ Rh film, deposited by direct current (dc) magnetron and radio frequency (rf) diode sputtering, respectively. The substrate was a (100) silicon wafer, with a thermal oxide layer of about $500 \mathrm{~nm}$ and a $\mathrm{Cu}$ buffer of $30 \mathrm{~nm}$. Both targets were equipped with screens to get the uniformity of the layer thickness better than $1 \%$. The base pressure was $5 \times 10^{-8}$ mbar and the Ar pressure was $10^{-2}$ mbar for dc magnetron $\left(\mathrm{Co}\right.$ and $\mathrm{Cu}$ ) and $5 \times 10^{-3}$ mbar rf diode (Rh) sputtering.

For the MSHG measurements we used the output of a Ti:Sapphire laser operating at a wavelength of $840 \mathrm{~nm}$ with a repetition rate of $82 \mathrm{MHz}$ and a pulse width of $100 \mathrm{fs}$. The 

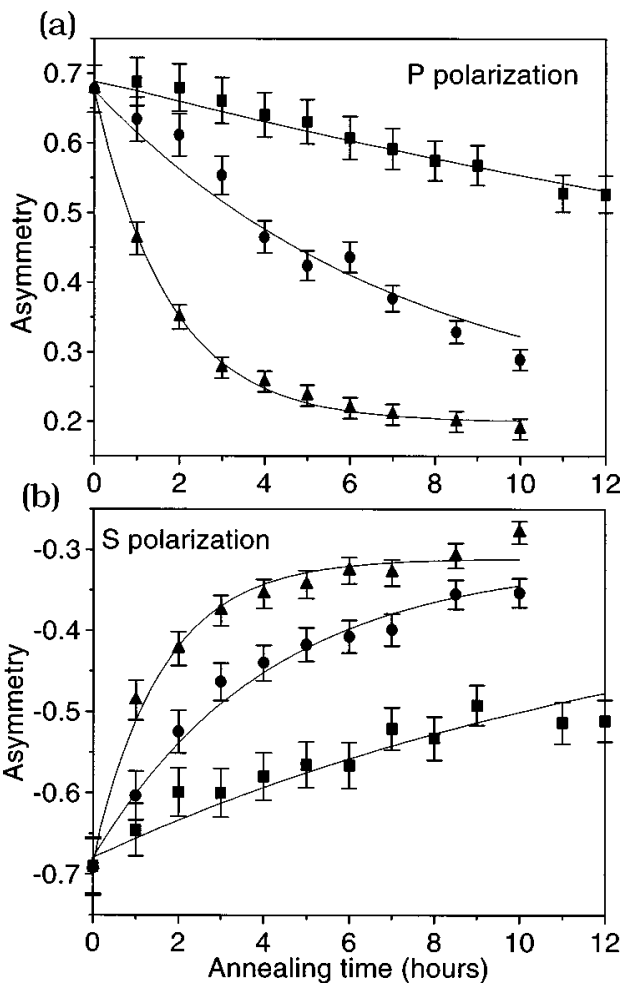

FIG. 1. (a) The asymmetry ( $P_{\text {in }} P_{\text {out }}$ polarization combination) as a function of annealing hours at different temperatures. Squares: $T=403 \mathrm{~K}$; circles: $T=433 \mathrm{~K}$; triangles: $T=453 \mathrm{~K}$. The lines are exponential fits. (b) The same but now for the $S_{\text {in }} P_{\text {out }}$ polarization combination.

average power at the sample was about $250 \mathrm{~mW}$, with a spot diameter of approximately $200 \mu \mathrm{m}$. The experiments were done in the transversal configuration, i.e., the magnetization $\mathbf{M}$ was in the plane of the sample and perpendicular to the optical plane of incidence. After proper filtering, the outcoming specular SH light was detected with a photomultiplier. We checked that for both $P_{\text {in }}$ and $S_{\text {in }}$ incoming light polarizations, the $\mathrm{SH}$ output was always strictly $P$ polarized $\left(P_{\text {out }}\right)$, in agreement with theory. ${ }^{8}$ As a magnetic signal, we measured the asymmetry $A$ [Eq. (3)]. The temperature of the sample holder could be varied between room temperature and $500 \mathrm{~K}$.

Figures 1(a) and 1(b) show that $A$ exhibits an exponential decay as a function of annealing time, with a decreasing decay time for increasing temperatures. The solid curves, in Figs. 1(a) and 1(b) are the least-square fits to a single exponential function, $A \sim \exp (-\alpha t)$. The simultaneously measured linear MOKE signal stayed constant, revealing no change in the bulk magnetic properties of the Co film. This observation was supported by vibrating sample magnetometer (VSM) measurements.

Taking into account the thickness of the Co layer (50 $\mathrm{nm}$ ) and the linear optical parameters of the materials involved, it can easily be shown that the MSHG signal is primarily due to the $\mathrm{Rh} / \mathrm{Co}$ interface. Together these results indicate that the temperature dependence of the MSHG signal probes a changing $\mathrm{Co} / \mathrm{Rh}$ interface contribution.

By plotting the thus obtained change in the interface magnetization in an Arrhenius plot (Fig. 2), we can obtain the activating energy for the changes at the $\mathrm{Co} / \mathrm{Rh}$ interface. A least-square fits of the data to $\alpha=\alpha_{0} \exp \left(-E_{\text {act }} / k_{B} T\right)$ Downloaded 16 Jun 2008 to 131.174.20.161. Redistribution subje

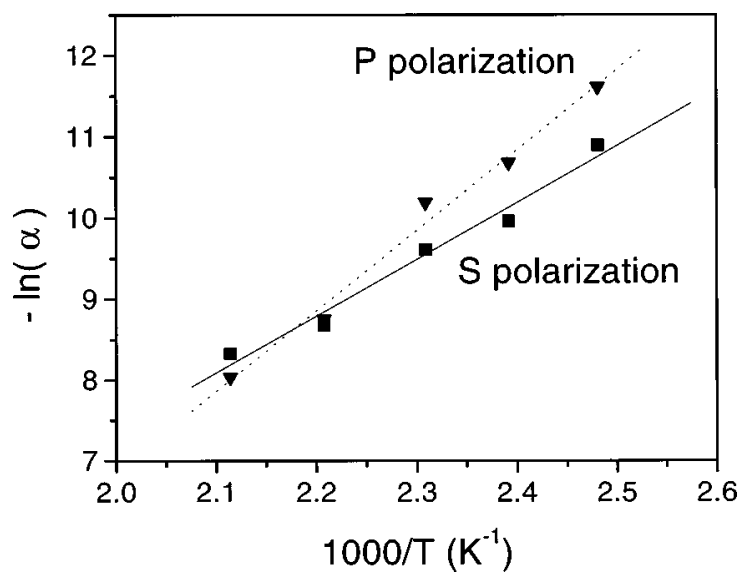

FIG. 2. The Arrhenius plot obtained from the results of Fig. 1.

gives an activation energy for $P$ polarization of $E_{\text {act }}=0.85$ $\pm 0.1 \mathrm{eV}$. A similar analysis for the $S$ polarization input yielded $E_{\text {act }}=0.6 \pm 0.1 \mathrm{eV}$. This apparent difference does not mean that there are two activation barriers, but must be a result of the fact that $P$ and $S$ polarizations can have different probing depths. ${ }^{9}$ Though MSHG is intrinsically interface sensitive due to the symmetry breaking, the nonlinear optical response for $S$-polarized input can be affected by a deeper layer ( $\sim 10-50 \AA$ ) than that for $P$-polarized light. Assuming the bulk contribution to be unaffected (or much less affected) by anything that happens at the interface, then this effectively reduces the interface sensitivity of MSHG for this polarization. To correct for this effect, we must assume the bulk contribution to be about $15 \%$.

To see whether the magnetic or the crystallographic $\chi$ components are changing with temperature, we measured the angle of incidence dependence of the MSHG signal for 433 and $453 \mathrm{~K}$. With the help of a transfer matrix technique (as described in Ref. 10) it is possible to separate the different interfaces and the different $\chi$ components. Out of these fits it followed that both the magnetic as well as the crystallographic $\chi$ components are changing with temperature. Their

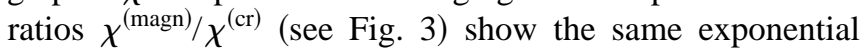
decays as are shown in Fig. 1, with the same activation energies.

The question now is what is the origin of this changing asymmetry? At this stage we can only speculate about its

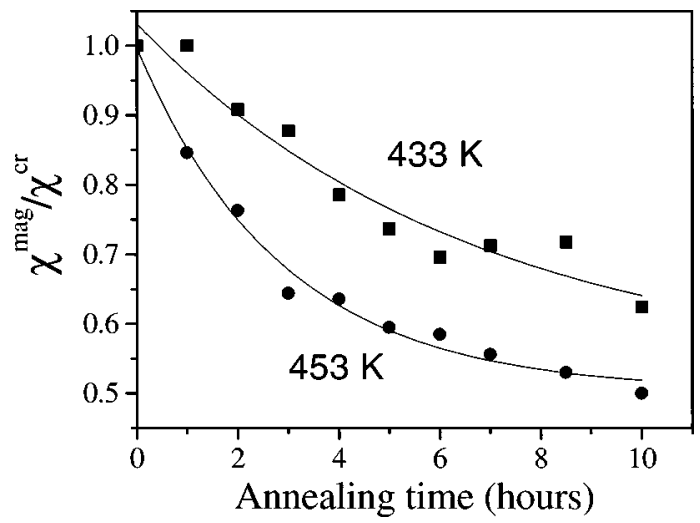

FIG. 3. The ratio between $\chi^{(\mathrm{magn})} / \chi^{(\mathrm{cr})}\left(S_{\mathrm{in}} P_{\text {out }}\right.$ polarization combination) as a function of annealing hours at different temperatures. The lines are exponential fits.

o AIP license or copyright; see http://apl.aip.org/apl/copyright.jsp 
exact origin, though we can rule out some possibilities. First of all, our VSM measurements, which were done on samples which had been annealed for $24 \mathrm{~h}$ at $433 \mathrm{~K}$, show that $2 \mathrm{~nm}$ of $\mathrm{Rh}$ is a good cap layer that prevent Co from oxidation. Secondly, at higher temperatures, $\mathrm{Co}$ and $\mathrm{Rh}$ interdiffuse. From Rutherford backscattering (RBS) data we know that mostly $\mathrm{Rh}$ is diffusing into Co and not vice versa. However, it is also known that the magnetic moment of the Co is not affected by the presence of $\mathrm{Rh}^{11}$ So the observed reducing magnetic anisotropy can be related to a decrease of the Co concentration at the interface. The found activation energy of $0.85 \mathrm{eV}$ then should correspond to the $\mathrm{Co} / \mathrm{Rh}$ interdiffusion, which looks quite reasonable and is of the same order of magnitude as those found for $\mathrm{Rh} / \mathrm{Au}(0.93 \mathrm{eV})^{12}$ and for $\mathrm{Co} / \mathrm{Pt}(0.8 \mathrm{eV}) .{ }^{13}$ Interdiffusion also explains why for $S$ polarization the apparent activation energy is lower. Because interdiffusion will only have a minor effect on the bulk response, the $S$ polarization measures an average between a changing interface and a constant bulk contribution. Because of the relative phase of the surface and bulk contributions, the bulk signal actually subtracts from the surface signal, in the $S$ geometry, yielding a smaller signal and larger apparent changes in the asymmetry of this signal, for the same amount of structural change at the interface; Thus the smaller activation energy in the $S$ configuration.

In conclusion, we have shown that the new nonlinear magneto-optical technique MSHG has an extremely high sensitivity to the quality of the interfaces and that it is possible to measure in situ the changes in the interface magnetization. From the obtained data we calculated the activation energy for the changes at the $\mathrm{Co} / \mathrm{Rh}$ interface and found it to be about $0.9 \mathrm{eV}$. The apparent difference in activation energy derived from the $S$ - or $P$-polarized input light can be explained by the difference in probing depth. After decompos- ing the total MSHG response into different contributions we found that both the magnetic and the crystallographic $\chi$ components are changing with the temperature but the ratio between the two behaves in exactly the same way as the asymmetry of the SH signal.

Part of this work was supported by the Stichting Fundamenteel Onderzoek der Materie (FOM) and by the EU through fellowships ERBCH-BGCT930444, ERBCHRXCT940563, and the TMR network ERBFMRXCT960015 (NOMOKE). One of the authors (F.M.) acknowledges financial support and the hospitality of Siemens AG. during his stay at Erlangen.

${ }^{1}$ P. Grünberg, R. Schreiber, Y. Pang, M. B. Brodsky, and H. Sowers, Phys. Rev. Lett. 57, 2442 (1986).

${ }^{2}$ S. S. P. Parkin, N. More, and K. P. Roche, Phys. Rev. Lett. 64, 2304 (1990).

${ }^{3}$ M. N. Baibich, J. M. Broto, A. Fert, F. Nguyen Van Dau, F. Petroff, P. Etienne, G. Creuzet, A. Friederich, and J. Chazelas, Phys. Rev. Lett. 61, 2472 (1988).

${ }^{4}$ J. Reif, J. C. Zink, C.-M. Schneider, and J. Kirschner, Phys. Rev. Lett. 67, 2878 (1991).

${ }^{5}$ H. A. Wierenga, W. de Jong, M. W. J. Prins, Th. Rasing, R. Volmer, A. Kirilyuk, H. Schwabe, and J. Kirschner, Phys. Rev. Lett. 74, 1462 (1995).

${ }^{6}$ B. Koopmans, M. Groot Koerkamp, Th. Rasing, and H. van den Berg, Phys. Rev. Lett. 74, 3692 (1995).

${ }^{7}$ R. Stolle, K. J. Veenstra, F. Manders, N. Persat, H. van den Berg, and Th. Rasing, Phys. Rev. B 55, R4925 (1997).

${ }^{8}$ R.-P. Pan, H. D. Wei, and Y. R. Shen, Phys. Rev. B 39, 1129 (1989).

${ }^{9}$ A. V. Petukhov and A. Liebsch, Surf. Sci. 334, 195 (1995).

${ }^{10}$ H. A. Wierenga, M. W. J. Prins, and Th. Rasing, Physica B 204, 281 (1995).

${ }^{11}$ S. Zoll, A. Dinia, D. Stoeffler, M. Gester, H. A. M. van den Berg, and K. Ounadjela, Europhys. Lett. 39, 323 (1997).

${ }^{12}$ W. J. DeBonte, J. M. Poate, C. M. Melliar-Smith, and R. A. Levesque, J. Appl. Phys. 46, 4284 (1975).

${ }^{13}$ P. C. McIntyre, D. T. Wu, and M. Nastasi, J. Appl. Phys. 81, 637 (1997). 